\title{
ESCALA DE DIFICULTADES EN REGULACIÓN EMOCIONAL (DERS): ANÁLISIS FACTORIAL EN UNA MUESTRA COLOMBIANA
}

\author{
Amanda M. Muñoz-Martínez* Rochy M. Vargas \\ Juan Sebastián Hoyos-González \\ Fundación Universitaria Konrad Lorenz, Bogotá - Colombia
}

Recibido, septiembre 11/2015

Concepto evaluación, noviembre 5/2015

Aceptado, diciembre 8/2015
Referencia: Muñoz-Martínez. A.M., Vargas, R.M. \& HoyosGonzález, J.S. (2016). Escala de Dificultades en Regulación Emocional(DERS): Análisis Factorial en una Muestra Colombiana. Acta Colombiana de Psicología, 19(1), 233-244. DOI: 10.14718/ ACP.2016.19.1.10

Resumen

La Escala de Dificultades en la Regulación Emocional (DERS, por sus siglas en inglés) es un instrumento diseñado para medir la desregulación emocional. El presente estudio buscó identificar la consistencia de los factores incluidos en la DERS, y la relevancia de los ítems en cada uno de ellos. Los participantes fueron 251 estudiantes, $68 \%$ mujeres, de una universidad en Bogotá-Colombia. En esta investigación se evaluaron los componentes de la DERS: (a) no-aceptación, (b) metas, (c) impulsividad, (d) estrategias, (e) consciencia, y (f) claridad. El Análisis Factorial mostró que los ítems de la DERS se reunían en dos factores principales en lugar de seis, y la reducción de datos demostró que 15 de los 36 ítems originales contribuyeron significativamente a la varianza. Se recomienda incrementar las investigaciones en contextos de la salud, y con población clínica y no clínica en Colombia para validar la DERS, y desarrollar un puntaje total de esta escala.

Palabras clave: desregulación emocional, análisis factorial, investigación instrumental.

\section{DIFFICULTIES IN EMOTION REGULATION SCALE (DERS): FACTOR ANALYSIS IN A COLOMBIAN SAMPLE}

\begin{abstract}
The Difficulties in Emotion Regulation Scale DERS) is an instrument designed to assess emotional dysregulation. The current study sought to identify the consistency of DERS factors, and items relevance for each one of them. Participants were 251 students, $68 \%$ female, from a university in Bogotá-Colombia. This research assessed the DERS factors: (a) non-acceptance, (b) goals, (c) impulse, (d) strategies, (e) awareness, and (f) clarity. Factor Analysis found that the DERS items are gathered in two main factors rather than six, and data reduction demonstrated that only 15 of the 36 items in the original scale contributed significantly to factors variance. It is recommended extending the research to health contexts, and to clinical and non-clinical population in Colombia to validate the DERS and develop a total score for the scale.

Key words: emotional dysregulation, factor analysis, instrumental study.
\end{abstract}

* Carrera 9a No. 69-27, Bogotá, Colombia. Tel: (+1) 7752236736 amandam.munozm@konradlorenz.edu.co Los autores agradecen al profesor Juan Carlos Rincón (Fundación Universitaria Konrad Lorenz) por el apoyo en el análisis estadístico. El presente trabajo fue desarrollado dentro del Proyecto de investigación "Análisis de la regulación emocional y los factores contextuales funcionales relacionados a esto en estudiantes universitarios: algunas guías de intervención” financiado por la Fundación Universitaria Konrad Lorenz del 2012 al 2013. (Código del proyecto: 95102131). 


\title{
ESCALA DE DIFICULDADES DE REGULAÇÃO EMOCIONAL (DERS): ANÁLISE FATORIAL NUMA AMOSTRA COLOMBIANA
}

\author{
Resumo
}

\begin{abstract}
A Escala de Dificuldades de Regulação Emocional (DERS, por sua sigla em inglês) é um instrumento desenhado para medir a desregulação emocional. O presente estudo buscou identificar a consistência dos fatores incluídos na DERS e a relevância dos itens em cada um deles. Os participantes foram 251 estudantes, 68\% mulheres, de uma universidade em Bogotá (Colômbia). Nesta pesquisa, avaliaram-se os componentes da DERS: 1) não aceitação; 2) metas; 3) impulsividade; 4) estratégias; 5) consciência e 6) clareza. A análise fatorial mostrou que os itens da DERS se reuniam em dois fatores principais em lugar de seis, e a redução de dados mostrou que 15 dos 36 itens originais contribuíram significativamente para a variação. Recomendase aumentar o número de pesquisas em contextos da saúde e com população clínica e não clínica na Colômbia para validar a DERS e desenvolver uma pontuação total dessa escala.

Palavras-chave: desregulação emocional, análise fatorial, pesquisa instrumental.
\end{abstract}

La Desregulación Emocional (DE) y su relación con problemas psicológicos ha llamado la atención de investigadores aplicados y clínicos. Estudios en contextos de salud mental han mostrado cómo diferentes problemas psicológicos implican dificultades emocionales (Angst, Angst \& Stassen, 1999; Beck, Kovacs \& Weissman, 1979; Calvo, Sánchez \& Tejada, 2003; Fenton, McGlashon, Victor \& Blyler, 1997; Goodman, Carpenter, Tang, Goldstein, Avedon, Fernández, et al., 2014; Hirshfeld \& Rusell, 1997; Jacobs, 1999; Lavender, Wonderlich, Engel, Gordon, Kaye \& Mitchell, 2015; Law \& Chapman, 2015; Little, Welsh, Darling \& Culpepper, 2015; Neacsiu, Eberle, Kramer, Wiesmann \& Linehan, 2014; Mann, Oquendo, Underwood \& Arango,1999; Masi, Muratori, Manfredi, Pisano \& Milone, 2015; Moscicki, 1995; Ministerio de la Protección Social, 2005; PosadaVilla, Aguilar-Gaxiola, Magaña \& Gómez, 2004; Powell, Geddes, Hawton, Deek \& Goldcare, 2000; Rich, Dhosse, Ghani \& Isacsson, 1998; Ridings \& Lutz-Zois, 2014; World Health Organization, 2012; Zutphen, Siep, Jacob, Goebel \& Arntz, 2015). La investigación ha demostrado una relación entre la Desregulación Emocional, las autolesiones y las conductas suicidas (Anestis, Bagge, Tull \& Joiner, 2011; Gratz, Tull, Barush, Bornovalova \& Lejuez, 2008; Goodman, Carpenter, Tang, Goldstein, Avedon, Fernandez, et al., 2014; Gratz, 2007; Law \& Chapman, 2015; Masi, Muratori, Manfredi, Pisano \& Milone, 2015; Neacsiu, Eberle, Kramer, Wiesmann \& Linehan, 2014; Rajappa, Gallagher \& Miranda, 2012; Ridings \& Lutz-Zois, 2014; Sánchez \& Tejada, 2003; Zutphen, Siep, Jacob, Goebel \& Arntz, 2015).

En los contextos de la salud, los investigadores han encontrado que la DE correlaciona con el uso de sustancias (Bonn-Miller, Vujanovic \& Zvolensky, 2008), el malestar psicológico (Aldea \& Rice, 2006), y el deterioro en la calidad de vida de los pacientes y sus familias (Wehmeier, Schacht \& Barkley, 2010). Por lo tanto, la Desregulación Emocional se ha convertido en un problema de salud pública, que necesita ser entendido y manejado para mejorar el bienestar de la población en general.

Aunque la DE cuenta con una amplia gama de aproximaciones conceptuales y metodológicas (Davies, Niles, Pitting, Arch \& Craske, 2015; Goodman, Carpenter, Tang, et al., 2014), carece de una definición precisa para poder diferenciarla de otros conceptos. Por ejemplo, algunos investigadores han definido la DE como un mecanismo de cambio (proceso), mientras que otros autores la han conceptualizado como una estrategia terapéutica (Lavender, Wonderlich, Engel, Gordon, Kaye \& Mitchell, 2015; Neacsiu, Eberle, Kramer, Wiesmann \& Linehan, 2014; Powers, Stevens, Fani, \& Bradley, 2015; Ridings \& LutzZois, 2014; Seligowski \& Orcutt, 2015; Weis, Gratz \& Lavender, 2015), lo que ha interferido con la investigación en este campo (Gratz, 2007; Vargas \& Muñoz-Martínez, 2013; Kököyei, Urbán, Reinhardt, Józan \& Demetrovics, 2014; Little, Welsh, Darling \& Culpepper, 2015; Masi, Muratori, Manfredi, Pisano \& Milone, 2015).

La definición de Linehan (1993) sobre Desregulación Emocional es la más aceptada entre las terapias cognitivoconductuales y las intervenciones conductuales. Linehan (1993) conceptualizó la DE como "una alta vulnerabilidad emocional para regular la emoción [...] así como un déficit en la habilidad de la modulación emocional" (p. 43). Aunque esta definición hace énfasis en las características morfológicas de las respuestas emocionalmente desreguladas, ignora aquellos factores que alteran las conductas emocionales (Cole, Michel \& Teti, 1994; Gratz, 2007). Así mismo, tal definición no explica la Regulación Emocional (RE), que es el centro de la Desregulación Emocional desde esa perspectiva. 
Seligowski y Orcutt (2015) han estado trabajando en el desarrollo de un modelo factorial de la Regulación Emocional para aclarar y evaluar este constructo basándose en sus características observables, en contraste con la perspectiva de Gross (1998, 1999), donde la regulación emocional se fundamenta en cuatro constructos teóricos (selección de situación, despliegue atencional, cambio cognitivo y modulación de la respuesta)

A pesar de la brecha en la definición de Regulación Emocional y Desregulación Emocional, los investigadores han seleccionado los principales elementos involucrados en ambos conceptos para llenar algunos vacíos en sus conceptualizaciones. Uno de los conceptos principales en RE y DE es el control emocional (Eisenberg, Cumberland \& Spinrad, 1998; Flett, Blankstein \& Obertynski, 1996; Garrido-Rojas, 2006). Varios instrumentos que evalúan la DE y la RE están basados en dicho concepto (Catanzaro \& Mearns, 1990; Salovey, Mayer, Goldman, Turvey \& Palfai, 1995). Estos instrumentos asumen que la poca habilidad para controlar las respuestas emocionales es el centro de la $\mathrm{DE}$, mientras que la modulación de respuestas emocionales es una habilidad central para regularse emocionalmente (Weis, Gratz \& Lavender, 2015).

Las habilidades de aceptación y consciencia también son conceptualizadas como elementos centrales para la RE y la DE. Por una parte, la aceptación es la habilidad de experimentar emociones sin intentar controlarlas o cambiarlas. Por lo tanto, hay un estrecho vínculo con la experiencia emocional, por lo que hace parte de las habilidades para regular la emoción. Por otro lado, Gratz y Roemer (1994) definen la consciencia como la habilidad de atender y entender respuestas emocionales. Sin embargo, hay algunas definiciones alternativas de consciencia desde una perspectiva analítica conductual. Por ejemplo, León (2006) define consciencia como una habilidad para experimentar la propia emoción, reconocerla y observarla. Catania (2004) define consciencia como la habilidad para relacionar el comportamiento con las características contextuales; comprende un repertorio amplio que involucra el darse cuenta de varias clases de respuesta, incluyendo los comportamientos emocionales. Sin embargo, el vínculo de la consciencia con la DE es confuso, ya que esta es vista como un repertorio general, que implica observar la relación entre cualquier tipo de comportamiento (emocional o no) y el contexto.

Para reducir la brecha en la definición de consciencia, Caycedo, Gutiérrez, Ascencio y Delgado (2005) plantearon que esta se relaciona con las habilidades de RE. Por esto, cuando los individuos reconocen las emociones, el contexto donde ocurren y otros repertorios conductuales emitidos para manejar las respuestas emocionales, ellos pueden modular eficazmente sus propios comportamientos. Esto significa que la consciencia puede ser un prerrequisito conductual para la RE, como una habilidad amplia e independiente (Weis, Gratz \& Lavender, 2015).

Basándose en las características principales que refiere la literatura sobre la Desregulación Emocional, Gratz y Roemer (2004) desarrollaron la Escala de Dificultades en Regulación Emocional (DERS, por sus siglas en inglés). Este instrumento busca medir varias características de la $\mathrm{DE}$, que de acuerdo con los autores está integrado por seis factores: (a) no aceptación de las respuestas emocionales (no-aceptación), (b) dificultades en conductas dirigidas a metas cuando se está alterado (metas), (c) dificultades para controlar comportamientos impulsivos cuando se está alterado (impulsividad), acceso limitado a estrategias de regulación emocional percibidas como efectivas (estrategias), (e) falta de consciencia emocional (consciencia), y (f) falta de claridad emocional (claridad).

La categoría de no-aceptación hace referencia a la reacción negativa a las respuestas emocionales de uno mismo y de otros. La dificultad en conductas dirigidas a metas implica que las emociones interfieren con una acción efectiva hacia una meta, mientras las personas están experimentando emociones negativas. La impulsividad se refiere a los problemas para controlar el propio comportamiento cuando se experimenta una emoción con alta intensidad. Consciencia es la categoría para medir las dificultades en reconocer y darse cuenta de sus propias emociones. El acceso limitado a estrategias de regulación emocional hace referencia a la pobre percepción de habilidades para modular las emociones. Finalmente, la claridad mide las dificultades para diferenciar las emociones mientras estas son experimentadas (Gratz \& Roemer, 2004).

Gratz y Roemer (2004) realizaron un Análisis Factorial Exploratorio (EFA) utilizando el método de extracción de componentes principales con una rotación oblicua promax, para evaluar la estructura factorial y la correlación de factores de la DERS; encontraron seis factores principales dentro de la DERS (ver arriba la descripción de los factores). La confiabilidad test-retest fue alta, $\alpha=.88, \mathrm{p}<0.01$, y se encontró una adecuada validez de constructo y predictiva. Sin embargo, algunos factores en la escala presentaron bajas correlaciones entre ellos. Finalmente, Gratz y Roemer (2004) sugirieron realizar investigaciones en diferentes contextos y poblaciones para mejorar el alcance y la validez externa de la escala.

Bardeen, Fergus y Orcutt (2012) realizaron un Análisis Factorial Confirmatorio (AFC) para ampliar los resultados obtenidos por Gratz and Roemer (2004). Encontraron que 
cinco de los seis factores de la DERS correlacionaron, excepto el de consciencia. Este último mostró una menor contribución a los factores en general de la escala. Bardeen, Fergus y Orcutt (2012) explicaron la baja correlación de consciencia con los otros factores, basándose en la definición adoptada en la DERS con respecto a este factor. Sin embargo, otros investigadores señalaron que es posible que la consciencia no comparta características con el constructo de Desregulación Emocional, a pesar de que puede hacer parte de las habilidades de RE (Vargas \& Muñoz-Martínez, 2013; Weis, Gratz \& Lavender, 2015).

Varios estudios han mostrado que la DERS es útil para evaluar dificultades emocionales en una variedad de trastornos psicológicos como: trastorno de estrés postraumático, trastorno límite de la personalidad, trastorno de ansiedad generalizada, uso de sustancias, trastorno de ataque de pánico y trastornos alimenticios (Fox et al., 2007; Gratz et al., 2008; Gratz \& Chapman, 2007; Gratz, Rosenthal, Tull, Lejuez \& Gunderson, 2006; Lavender et al., 2015; McDermott et al., 2009; Roemer et al., 2009; Tull, 2006; Tull, Stipelman, Salters-Pedneault \& Gratz, 2009), dolor crónico (Kököyei, Urbán, Reinhardt, Józan \& Demetrovics, 2014), y alexitimia (Ridings \& Lutz-Zois, 2014). También hay estudios que han demostrado la utilidad de la DERS para medir problemas transdiagnósticos que se relacionan con Desregulación Emocional (Neacsiu, Eberle, Kramer, Wiesmann \& Linehan, 2014) y dificultades para regular emociones positivas (Weis, Gratz \& Lavender, 2015).

Además, la DERS ha demostrado buenas propiedades psicométricas en muestras clínicas con diferentes edades (Gómez, Penelo \& De la Osa, 2014; Marin, Robles, GonzálezForteza \& Andrade, 2012; Staples \& Mohlman, 2012; Weinberg Klonky, 2009) y con diferentes poblaciones étnicas (Gómez, Penelo \& De la Osa, 2014; Guzmán-González, Garrido \& Leiva, 2014; Marin, Robles, González-Forteza \& Andrade, 2012).

La DERS ha sido validada en diferentes países: Turquía, Italia, España, Argentina, Chile y México. La validación realizada en Turquía (Ruganci \& Gençöz, 2010) mantuvo la estructura y método utilizados por Gratz y Roemer (2004) para evaluar la estructura factorial (método de componentes principales y la rotación oblicua promax). Ellos evaluaron la confiabilidad test-retest y la consistencia interna. Ruganci y Gençöz (2010) encontraron una estructura factorial de la DERS similar a la encontrada por Gratz y Roemer (2004). Sin embargo, no encontraron correlación entre consciencia y los otros factores dentro de la escala.

Giromini, Velotti, de Campora, Bonalume y CesareZavattini (2012) validaron la DERS en Italia. Encontraron una estructura factorial similar (seis factores) a la de Gratz y Roemer (2004) y Ruganci y Gençöz (2010). El índice de confiabilidad fue alto con los datos obtenidos en muestras clínicas y no clínicas.

Medrano y Trógolo (2014) adaptaron la DERS en Argentina, realizaron un EFA con una rotación promax y una estimación ponderada de mínimos cuadrados para establecer la estructura factorial de la escala. La versión argentina de la DERS tiene 28 ítems agrupados en seis factores (igual que la versión original), que explicaba el $50.79 \%$ de la varianza. El análisis del alpha de Cronbach fue realizado para cada factor (no aceptación $\alpha=.84$; metas $\alpha=.82$; impulsividad $\alpha=.87$; estrategias $\alpha=.54$; consciencia $\alpha=.71$; claridad $\alpha=.7)$, encontrando altos índices de confiabilidad en cinco de los seis factores, y demostrando validez concurrente al compararla con una escala de personalidad (International Personality Item Pool-IPIP-).

Hervás y Jódar (2008) adaptaron y validaron la DERS en España, evaluando la misma estructura factorial propuesta por quienes desarrollaron la DERS. De acuerdo con el análisis realizado por estos autores, algunos ítems se traslapaban entre los factores de impulsividad y estrategias. Por ello, reunieron las dos categorías y constituyeron un nuevo factor denominado descontrol. Después, cambiaron los nombres de las demás categorías. En el formato final de España, no-aceptación es rechazo cambiante, problemas con metas es interferencia, consciencia es desatención y claridad es confusión. Gómez, Penelo y De la Osa (2014) validaron la escala con 642 adolescentes de 12 a 18 años en España. El análisis factorial indicó una solución de seis factores y una medida de invariancia estricta entre sexos. La consistencia interna de todas las subescalas fue entre moderada y satisfactoria $(\alpha=.71-.88)$, con excepción de consciencia $(\alpha=.62)$.

Marín, Robles, González-Forteza y Andrade (2012) probaron las propiedades psicométricas del formato en español de la DERS (DERS-E) con una población no clínica de adolescentes en México. Los participantes fueron 455 estudiantes de secundaria de un colegio público (media de edad=13.1). Realizaron un Análisis Factorial Confirmatorio y en comparación con la versión original, no encontraron la estructura de seis factores utilizando los 36 ítems $\left(\mathrm{c}^{2}(6)=\right.$ 22339.4, $\mathrm{p} \leq .05$ ). A este respecto, desarrollaron un Análisis Factorial Exploratorio, encontrando un modelo de cuatro factores con 24 ítems; la DERS fue validada utilizando un CFA, $\mathrm{c}^{2}(230)=259.73, \mathrm{p}<.05$. La confiabilidad de las subescalas fue alta ( $\alpha=.68$ to .85 ) y la validez concurrente fue significativa ( $\mathrm{r}=.51$ to.76, $\mathrm{p} \leq .05)$.

En Chile, Guzmán-González, Garrido y Leiva (2014) realizaron un estudio probando la validez y la confiabilidad de la DERS-E en población chilena. Ellos realizaron 
el análisis con estudiantes universitarios (1018) y adultos (1161), con una muestra total de 2.179 personas. Los participantes completaron la DERS-E y el Cuestionario de Resultados (OQ-45.2, por sus siglas en inglés). Los resultados confirmaron las propiedades psicométricas de la DERS-E para medir dificultades en regulación emocional.

Herrera, Niño, Caycedo y Cortés (2008) realizaron el análisis de confiabilidad para la DERS $(\alpha=.90)$ en Colombia. Aunque encontraron un alto índice de confiabilidad, no realizaron un análisis factorial o de algún otro tipo para validar la escala. Los autores recomendaron ejecutar otros estudios con una población más amplia utilizando pruebas estadísticas robustas para validar la DERS en población colombiana.

En resumen, la DERS ha demostrado su validez y confiabilidad para medir la Desregulación Emocional en varios estudios con poblaciones diversas en diferentes países. Sin embargo, algunas investigaciones han mostrado diferencias en su estructura factorial y diferencias significativas con respecto a la correlación entre factores en la DERS. Por esto, es relevante realizar estudios para aclarar dichas diferencias y dar explicaciones al respecto. Varios estudios también han recomendado realizar un análisis por ítem para establecer la exactitud y precisión de los ítems en la DERS, estableciendo si los ítems en la escala son suficientes y necesarios para medir adecuadamente la Desregulación Emocional. Finalmente, la relación entre la Desregulación Emocional, los problemas psicológicos y las dificultades en salud plantea la importancia de contar con una medida valida y confiable que identifique estas dificultades en la población colombiana.

\section{MÉTODO}

\section{Participantes}

Los participantes ( $\mathrm{N}=761$ ) fueron reclutados por medio de correo electrónico o de forma personal en una universidad privada de Bogotá-Colombia. Los objetivos y fines del estudio fueron presentados durante el primer contacto con la población.

Después de la aleatorización, 251 participantes fueron seleccionados. Eran estudiantes de psicología (52\%, $\mathrm{N}=130)$, negocios $(34 \%, \mathrm{~N}=85)$, e ingeniería y matemáticas $(14 \%, \mathrm{~N}=36) .78 \%$ de los participantes eran mujeres y $32 \%$ eran hombres.

\section{Instrumentos}

Escala de Dificultades en Regulación Emocional (Difficulties in Emotion Regulation Scale., DERS). Este estudio analizó el formato de la DERS de Herrera, Niño, Caycedo y Cortés (2008) que cuenta con un alto índice de confiabilidad $(\alpha=.90)$. A pesar de que aplicaron los ítems adaptados por Hervás y Jodár (2008), mantuvieron la estructura factorial y el nombre de las subescalas de Gratz y Roemer (2004).

La escala de autoinforme consiste en 36 ítems reunidos en seis factores: a) no aceptación de respuestas emocionales (no-aceptación), (b) dificultades en conductas dirigidas a metas cuando se está alterado (metas), (c) dificultades en controlar conductas impulsivas cuando se está alterado (impulsividad)), (d) acceso limitado a estrategias de regulación emocional percibidas como efectivas (estrategias), (e) falta de consciencia emocional (consciencia), y (f) falta de claridad emocional (claridad). El análisis original de la DERS reveló una buena consistencia interna $(\alpha=.88)$ y tiene validez de constructo y predictiva.

Procedimiento

Después del reclutamiento, los participantes aptos fueron completamente informados de forma verbal y escrita sobre los objetivos y el manejo de los datos; se les dio un consentimiento informado de acuerdo con el código ético de la American Psychological Association (APA), el cual describía los propósitos del estudio y la naturaleza confidencial de la información.

Debido a las diferencias en la estructura factorial de la DERS reportadas por algunos estudios (Marin et al., 2012), con respecto a los factores originales, donde los investigadores usaron una rotación oblicua (p.e. promax) un Análisis Factorial Exploratorio con una rotación ortogonal (varimax) fue utilizado para probar la estructura factorial en una población colombiana. El análisis de los datos determinó la distribución natural de los ítems sin una estructura factorial preestablecida a través de la rotación ortogonal, lo que probó la distribución natural de los ítems dentro de los factores, manteniendo independencia estadística entre ellos. Lo anterior hace que los factores no tengan por qué ajustarse a las suposiciones teóricas de los autores, lo que sucede con una rotación oblicua donde se asume un número específico de factores antes de iniciar el análisis (Brown, 2009). Además, se realizó una reducción factorial de datos para identificar la contribución de los ítems al evaluar las dificultades en la Regulación Emocional utilizando la DERS.

\section{RESULTADOS}

La estructura factorial de la DERS y la reducción de datos de los ítems fueron probados con un Análisis Factorial Exploratorio. La prueba de Kolmogrov-Smirnov para normalidad fue significativa $(p=.000)$ y la distribución fue positivamente asimétrica (1.41) para todos los factores en la DERS. No obstante, dado que el análisis factorial es una prueba estadísticamente robusta fue posible realizar 
el análisis de datos aun cuando estos no estaban normalmente distribuidos.

Análisis factorial de la estructura factorial de la DERS.

Para probar la estructura factorial original de la DERS se realizó un AFE. La prueba Kaiser-Meyer-Olkin (KMO) fue de .83, y la prueba de Bartlett de esfericidad fue significativa, $\mathrm{c}^{2}(15)=530.85, p=.000$.

El AFE, utilizando una rotación varimax, mostró que el $54 \%$ de la varianza fue explicada por el factor
1 y el 15\% por el factor 2. Sin embargo, los análisis no mostraron independencia entre los seis factores que comprendían la DERS. La matriz de componentes principales reveló que los factores no-aceptación, metas, impulsividad, estrategias y claridad contribuyeron significativamente al factor 1 , mientras que consciencia contribuyó de una forma importante al factor 2 . Ningún otro factor estuvo relacionado con el factor 2 (Véase Tabla 1).

Tabla 1.

Análisis de componentes principales con rotación ortogonal y la varianza explicada de la estructura factorial de la DERS

\begin{tabular}{|c|c|c|c|c|c|c|}
\hline & \multicolumn{6}{|c|}{ Componentes } \\
\hline & 1 & 2 & 3 & 4 & 5 & 6 \\
\hline F1 DERS - No aceptación & .714 & -.281 & .581 & .000 & .221 & .155 \\
\hline F2 DERS - Metas & .741 & -.252 & -.382 & .373 & .315 & -.057 \\
\hline F3 DERS - Impulsividad & .790 & -.14 & -.322 & -.406 & -.093 & .283 \\
\hline F4 DERS - Consciencia & .518 & .80 & .015 & -.134 & .279 & -.050 \\
\hline F5 DERS - Estrategias & .847 & -.17 & .068 & -.201 & -.175 & -.422 \\
\hline F6 DERS - Claridad & .764 & .30 & .074 & .371 & -.412 & .121 \\
\hline$\%$ of Varianza & 54.19 & 15.21 & 9.96 & 8.33 & 7.24 & 5.06 \\
\hline Varianza Total & 3.252 & .913 & .598 & .500 & .435 & .303 \\
\hline
\end{tabular}

Nota: Las contribuciones en negrita son valores mayores a .40 y son conservados para ese factor. Los valores subrayados indican contribuciones múltiples en dos factores.

Las correlaciones entre los factores de la DERS fueron significativas, con excepción de consciencia y no-aceptación, y consciencia y metas. No obstante, impulsividad y estrategias fueron los únicos factores que tuvieron una alta correlación significativa (.60) (Véase Tabla 2).

Tabla 2.

Correlaciones entre factores de la DERS con la estructura factorial original

\begin{tabular}{|c|c|c|c|c|c|c|}
\hline Factor & No aceptación & Metas & Impulsividad & Consciencia & Estrategias & Claridad \\
\hline No aceptación & - & & & & & \\
\hline Metas & $.20 * *$ & - & & & & \\
\hline Impulsividad & $.37 * *$ & $.23 * *$ & - & & & \\
\hline Consciencia & .11 & .12 & $.22 * *$ & - & & \\
\hline Estrategias & $.37 * *$ & $.18 * *$ & $.60 * *$ & $.28 * *$ & - & \\
\hline Claridad & $.22 * *$ & $.24 * *$ & $.25^{* *}$ & $.33 * *$ & $.30 * *$ & - \\
\hline
\end{tabular}

Nota: $* * \mathrm{p}<0.01$ 
Análisis Factorial Exploratorio (AFE) para los items de la DERS

La prueba KMO y de esfericidad fueron realizadas para evaluar los ítems de la DERS. La medida de adecuación muestral fue de .87 , por encima del valor recomendado de .6 , y la prueba de Bartlett de esfericidad fue significativa, $c^{2}(630)=4191.56, p=.000$. Las diagonales de la matriz de correlación anti-imagen estuvieron por encima de .65, apoyando la inclusión de cada ítem en el análisis factorial (Véase Tabla 3). Dados estos indicadores totales, el análisis factorial fue realizado con los 36 ítems.

Tabla 3.

Adecuación de muestreo a través de medidas de correlaciones

\begin{tabular}{|c|c|}
\hline Item & Correlación Anti-imagen \\
\hline 1 & .91 \\
\hline 2 & .65 \\
\hline 3 & .84 \\
\hline 4 & .84 \\
\hline 5 & .88 \\
\hline 6 & .78 \\
\hline 7 & .89 \\
\hline 8 & .68 \\
\hline 9 & .86 \\
\hline 10 & .88 \\
\hline 11 & .88 \\
\hline 12 & .82 \\
\hline 13 & .89 \\
\hline 14 & .87 \\
\hline 15 & .87 \\
\hline 16 & .91 \\
\hline 17 & .81 \\
\hline 18 & .90 \\
\hline 19 & .88 \\
\hline 20 & .74 \\
\hline 21 & .82 \\
\hline 22 & .73 \\
\hline 23 & .86 \\
\hline
\end{tabular}

Continuación tabla 3

\begin{tabular}{cc}
\hline Item & Correlación Anti-imagen \\
\hline 24 & .89 \\
25 & .94 \\
26 & .88 \\
27 & .92 \\
28 & .93 \\
29 & .88 \\
30 & .90 \\
31 & .86 \\
32 & .86 \\
33 & .91 \\
34 & .78 \\
35 & .90 \\
36 & .88 \\
\hline
\end{tabular}

Las comunalidades fueron inferiores a .5 para cuatro de los ítems. Asimismo, algunos ítems contribuyeron a más de un factor o tuvieron una baja contribución en otros factores, de acuerdo con la carga del factor (por debajo de .4) (Véase Tabla 4).

\section{Tabla 4.}

Carga de los factores y comunalidades basados en el análisis de componentes principales con una rotación varimax para 36 items de la DERS $(\mathrm{N}=251)$

\begin{tabular}{cccc}
\hline & Factor 1 & Factor 2 & Comunalidades \\
\hline 1 & .53 & .42 & .55 \\
2 & & .41 & .72 \\
3 & .38 & & .48 \\
4 & .41 & .34 & .54 \\
5 & .54 & & .65 \\
6 & & .61 & .66 \\
7 & .55 & .39 & .66 \\
8 & & .35 & .68 \\
9 & .51 & & .68 \\
10 & .48 & .32 & .55 \\
11 & .52 & -.42 & .66 \\
12 & .53 & -.36 & .71 \\
\hline
\end{tabular}


Continuación tabla 4

\begin{tabular}{|c|c|c|c|}
\hline & Factor 1 & Factor 2 & Comunalidades \\
\hline 13 & .57 & & .64 \\
\hline 14 & .67 & & .75 \\
\hline 15 & .64 & & .67 \\
\hline 16 & .65 & & .61 \\
\hline 17 & & .39 & .49 \\
\hline 18 & .60 & & .73 \\
\hline 19 & .66 & & .74 \\
\hline 20 & & & .65 \\
\hline 21 & .61 & & .75 \\
\hline 22 & .36 & .38 & .75 \\
\hline 23 & .56 & & .56 \\
\hline 24 & .44 & & .46 \\
\hline 25 & .63 & -.35 & .7 \\
\hline 26 & .61 & & .72 \\
\hline 27 & .70 & & .68 \\
\hline 28 & .67 & & .65 \\
\hline 29 & .62 & -.33 & .67 \\
\hline 30 & .68 & -.31 & .69 \\
\hline 31 & .63 & & .67 \\
\hline 32 & .63 & & .75 \\
\hline 33 & .63 & & .52 \\
\hline 34 & & .44 & .52 \\
\hline 35 & .49 & & .52 \\
\hline 36 & .46 & & .49 \\
\hline
\end{tabular}

Nota: Las cantidades de los factores presentan contribuciones por el factor 1 y el factor 2 .

En el análisis de componentes principales se encontró que los ítems se distribuyeron en nueve factores en los valores Eigen iniciales. Sin embargo, solo el primer y segundo factor contribuyeron significativamente a la varianza (Véase Figura 1). El primer factor explicó el $28 \%$ de la varianza y el segundo factor el $7 \%$ de la varianza. Los otros factores contribuyeron por debajo del $6 \%$ cada uno, siendo menos representativos (Véase Tabla 5).
Tabla 5.

Porcentaje de la varianza explicada

\begin{tabular}{ccc}
\hline & Varianza Total & \% de Varianza \\
\hline F1 & 10.21 & 28.37 \\
F2 & 2.71 & 7.52 \\
F3 & 2.22 & 6.17 \\
F4 & 1.73 & 4.80 \\
F5 & 1.48 & 4.12 \\
F6 & 1.20 & 3.32 \\
F7 & 1.19 & 3.30 \\
F8 & 1.09 & 3.01 \\
F9 & 1.00 & 2.80 \\
\hline
\end{tabular}

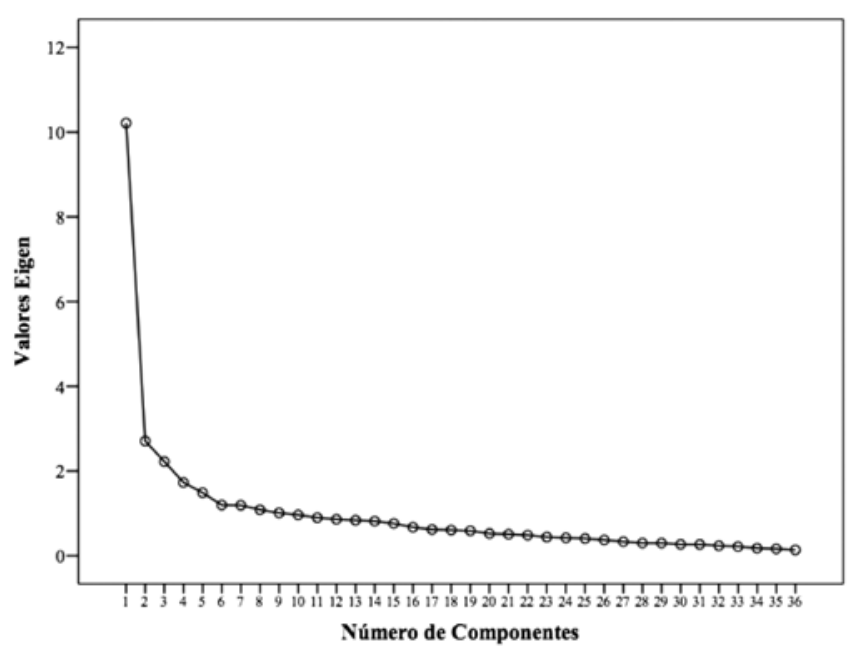

Figura 1. Contribución de los ítems a la estructura factorial de la DERS

La reducción de los datos, utilizando múltiples AFE, se realizó para eliminar los ítems que no contribuían a una estructura factorial simple y fallaron en cumplir con los criterios de: tener una carga mínima de .5 o por encima, y no tener cargas compartidas de .3 o por encima (Véase Tabla 4). Quince ítems constituyen el formato final de la DERS, agrupados en dos factores principales (Véase Tabla 6). 
Tabla 6.

Formato final de la DERS (Escala en español-colombiana)

\begin{tabular}{ll}
\hline FACTORES & \multicolumn{1}{c}{ ÍTEMS } \\
\hline 1 (Estrategias) & 5. Tengo dificultad para encontrar el significado a mis sentimientos. \\
9. Yo me siento confundido acerca de cómo me siento. \\
13. Cuando estoy alterado, tengo dificultad para realizar el trabajo. \\
14. Cuando estoy molesto, quedo fuera de control \\
15. Cuando estoy alterado, creo que seguirá siendo así durante mucho tiempo. \\
16. Cuando estoy alterado, creo que voy a terminar sintiéndome muy deprimido. \\
18. Cuando estoy alterado, tengo dificultad para concentrarme en otras cosas. \\
19. Cuando estoy alterado, me siento fuera de control. \\
21. Cuando estoy alterado, me siento avergonzado de mí mismo por sentir de esa manera. \\
26. Cuando estoy alterado, yo tengo dificultades concentrándome. \\
27. Cuando estoy alterado, tengo dificultades controlando mis comportamientos. \\
28. Cuando estoy alterado, creo que no hay nada que pueda hacer para sentirme mejor. \\
32. Cuando estoy alterado, pierdo el control sobre mis conductas. \\
33. Cuando estoy alterado, encuentro difícil pensar en algo más. \\
6. Yo estoy atento a mis sentimientos (r).
\end{tabular}

Nota: $r=$ ítem con calificación invertida.

\section{DISCUSIÓN}

Los ítems de la DERS en población colombiana se distribuyeron en dos factores, en contraste con los resultados de los análisis de Gratz y Roemer (2004); Ruganci y Gençöz (2010); Giromini, Velotti, de Campora, Bonalume, y Cesare-Zavattini (2012), y Medrano y Trogólo (2014). Los resultados de este estudio son similares a los encontrados por Marín, Robles, González-Forteza y Andrade (2012), quienes realizaron un AFC de la estructura factorial de la DERS, y al no encontrar la misma estructura factorial, hicieron un AFE, identificando un nuevo modelo de cuatro factores para la DERS en México. Las similitudes entre las investigaciones pueden estar relacionadas con el haber realizado un AFE para evaluar la estructura factorial de la DERS independiente de los factores preestablecidos en estudios anteriores.

Debido a que la estructura factorial y el número de ítems en el presente estudio cambió notablemente con respecto al formato original de la DERS, se recomienda probar este nuevo modelo realizando un AFC con una muestra más amplia en población colombiana. También se sugiere validar la escala con adultos y adolescentes en condiciones clínicas y no clínicas para estandarizar el instrumento. Puesto que el segundo factor se encuentra compuesto por un solo ítem, se recomienda examinar la utilidad del segundo factor para medir la Desregulación Emocional. Para probar la idoneidad del segundo factor, se necesita incluir nuevos ítems en la escala dentro de este factor para que se puedan hacer inferencias más fuertes al respecto. Incluso se puede considerar eliminar el factor de consciencia de la DERS, ya que parece ser un constructo independiente a la DE (Ver discusión abajo).

Los presentes resultados también son similares a los encontrados por Bardeen, Fergus y Orcutt (2012) donde efectuaron un AFC. Los dos estudios revelaron independencia del factor de consciencia con respecto a los otros componentes de la DERS. Así, el principal factor (1) está integrado por ítems diseñados para medir metas, impulsividad, estrategias, claridad, y no-aceptación, especialmente 
aquellos que evalúan las dificultades en las estrategias, y el segundo (2) obedeció solo al factor de consciencia.

La independencia de consciencia con respecto a los otros factores podría estar relacionada con entender y atender las emociones (Gratz \& Roemer, 2004). Otros autores refieren que los problemas con la consciencia ocurren cuando los individuos luchan con la identificación y descripción de la variedad de eventos en contexto (Stewart, Villatte \& McHugh, 2012). La consciencia también es conceptualizada como un repertorio amplio a través del cual las personas vinculan su propio comportamiento (e.j.. respuestas emocionales) con otros eventos (Catania, 2007; Caycedo, Gutiérrez, Ascencio \& Delgado, 2005; Dymond \& Barnes, 1997; León, 2006; Stewart, Villatte \& McHugh, 2012). Por esto, la consciencia no sería un repertorio enfocado exclusivamente en observar la relación entre las respuestas emocionales y el contexto, sino que incluye relacionar cualquier tipo de comportamiento con el ambiente que influye en éste; por lo tanto, consciencia es un repertorio conductual independiente que implica darse cuenta de sus propias acciones sin alterarlas, regularlas o modularlas. Esto da una mirada de la consciencia como un factor independiente de los constructos de regulación o desregulación (Weis, Gratz \& Lavender, 2015).

Las diferencias en la estructura factorial de la DERS en varios países también podrían estar relacionadas con cuestiones de diversidad cultural. Vale la pena llevar a cabo estudios que permitan identificar características de RE y DE en diferentes países para establecer si dichas diferencias corresponden a los problemas en la escala 0 están vinculados con características culturales.

Una de las limitaciones en este estudio es la distribución de la población, que restringe la generalización de los resultados. Usualmente las muestras de estudiantes no tienen una distribución normal y tienden a ser sesgadas, como en este estudio, por lo cual se recomienda llevar a cabo otra investigación en poblaciones más amplias, tanto clínicas como no clínicas, para establecer la ejecución de la DERS con muestras distribuidas normalmente. Además, no se realizaron otras pruebas para estimar la validez de la DERS. Por ello es importante ejecutar análisis multi-método y multi-rasgo para determinar la validez convergente y discriminante de la DERS en Colombia (Campbell \& Fiske, 1959).

Finalmente, para mejorar los resultados terapéuticos en contextos clínicos y de salud con consultantes que presentan dificultades emocionales, es relevante definir claramente los constructos de RE como un concepto independiente. Este último implicará el desarrollo de instrumentos enfocados en el bienestar emocional y repertorios funcionales con una aproximación enfocada en los progresos de los con- sultantes, más que en un enfoque centrado en la reducción de síntomas (Cloninger, 2009).

\section{REFERENCIAS}

Aldea, M. A., \& Rice, K. G. (2006). The role of emotional dysregulation in perfectionism and psychological distress. Journal of Counseling Psychology, 53, 498.

Anestis, M., Bagge, C. L., Tull, M. T. \& Joiner, T. E. (2011). Clarifying the role of emotion dysregulation in the interpersonal-psychological theory of suicidal behavior in an undergraduate sample. Journal of Psychiatric Research, 45, 603-611.

Angst, J., Angst, F. \& Stassen, H. (1999). Suicide Risk in patients with major depressive disorder. Journal of Clinical Psychiatry, 60 (supl.2), 57-62.

Bardeen, J. R., Fergus, T. A., \& Orcutt, H. K. (2012). An examination of the latent structure of the Difficulties in Emotion Regulation Scale. Journal of Psychopathology and Behavioral Assessment, 34, 382-392.

Beck, A., Kovacs, M. \& Weissman, A. (1979). Assessment of suicidal intent. The scale for suicide ideation. Journal of consulting and Clinical Psychology, 47, 343-352.

Bonn-Miller, M. O., Vujanovic, A. A., \& Zvolensky, M. J. (2008). Emotional dysregulation: association with copingoriented marijuana use motives among current marijuana users. Substance Use \& Misuse, 43, 1653-1665.

Brown, J. D. (2009). Choosing the right type of rotation in PCA and EFA. JALT Testing \& Evaluation SIG Newsletter, 13, 20-25.

Calvo, J., Sánchez, R.\& Tejada, P. (2003). Prevalencia y factores asociados a ideación suicida en estudiantes universitarios. Revista de Salud Pública, 5, 123-143.

Campbell, D. T., \& Fiske, D. W. (1959). Convergent and discriminant validation by the multitrait-multimethod matrix. Psychological bulletin, 56, 81-105.

Catanzaro, S. J. \& Mearns, J. (1990). Measuring generalized expectancies for negative mood regulation: Initial scale development and implications. Journal of Personality Assessment. 54, 546-563.

Caycedo, C., Gutiérrez, C., Ascencio, V. \& Delgado, A. (2005). Regulación emocional y entrenamiento en solución de problemas sociales como herramienta. Suma Psicológica. 12, 157-173.

Cloninger, C. R. (2006). The science of well-being: an integrated approach to mental health and its disorders. World Psychiatry, 5, 71.

Cole, P. M., Michel, M. K. \& Teti, L. O. (1994). The development of emotion regulation and dysregulation: A clinical perspective. In N. A. Fox (Ed.). The development of emotion regulation: Biological and behavioral considerations. Monographs of the Society for Research in Child Development. 59, 73-100. 
Davies, C., Niles, A., Pittig, A., Arch, J. \& Craske, M. (2015). Physiological and behavioral indices of emotion dysregulation as predictors of outcome from cognitive behavioral therapy and acceptance and commitment therapy for anxiety. Journal of Behavior Therapy and Experimental Psychiatry, 46, 35-43.

Dymond, S. \& Barnes, D. (1997). Behavior analytic approaches to self-awareness. The Psychological Record, 47, 181-200.

Eisenberg, N., Cumberland, A. \& Spinrad, T. L. (1998). Parental socialization of emotion. Psychological Inquiry, 9, 241273.

Fenton, W., McGlashon, T., Victor, B., \& Blyler, C. (1997). Symptoms, subtype and suicidality in patients with Schizophrenia spectrum disorders. American Journal of Psychiatry. 154, 199-204.

Flett, G. L., Blankstein, K. R., \& Obertynski, M. (1996). Affect intensity, coping styles, mood regulation expectancies, and depressive symptoms. Personality and Individual Differences, 20, 221-228

Fox, H. C., Axelrod, S.R., Paliwal, P., Sleeper. J. \& Sinha, R. (2007). Difficulties in emotion regulation and impulse control during cocaine abstinence. Drug and Alcohol Dependence. 89, 298-301.

Garrido-Rojas, L. (2006). Apego, emoción y regulación emocional. Implicaciones para la salud. Revista Latinoamericana de Psicología. 38, 493-507.

Giromini, L., Velotti, P., de Campora, G., Bonalume, L., \& Cesare Zavattini, G. (2012). Cultural adaptation of the difficulties in emotion regulation scale: Reliability and validity of an Italian version. Journal of Clinical Psychology, 68, 989-1007.

Gómez, I., Penelo, E. \& De la Osa, N. (2014). Estructura factorial e invarianza de la Escala de Dificultad en la Regulación Emocional (DERS) en adolescentes españoles. Psicothema, 26(3), 401-408.

Goodman, M., Carpenter, D., Tang, C., Goldstein, K., Avedon, J., Fernández, N., Mascitelli, K., Blair, N., New, A., Triebwasser, J., Siever, L. \& Hazlett, E. (2014). Dialectical behaviour therapy alters emotion regulation and amygdala activity in patients with borderline personality disorder. Journal of Psychiatric Research, 57, 108-116.

Gratz, K. L. \& Chapman, A. L. (2007). The role of emotional responding and childhood maltreatment in the development and maintenance of deliberated self-harm among male undergraduate. Psychology of men and masculinity. 8, 1-14.

Gratz, K. L. (2007). Targeting Emotional Dysregulation in the treatment of self-injury. Journal of Clinical Psychology: in session. 63, 1091-1103.

Gratz, K. \& Roemer, L. (2004) Multidimensional Assessment of Emotion Regulation and Dysregulation: Development, Factor Structure, and Initial Validation of the Difficulties in Emotion Regulation Scale. Journal of Psychopathology and Behavioral Assessment. 26, 41-54.

Gratz, K. L., Rosenthal, M. Z., Tull, M.T., Lejuez, C. W. \& Gunderson, J. G. (2006). An experimental investigation of
Emotional Dysregulation in Borderline Personality Disorder. Journal of Abnormal Psychology, 115, 850-855.

Gratz, K. L., Tull, M. T., Barush, D. E., Bornovalova, M. A. \& Lejuez, C. W. (2008). Factors associated with co-occurring borderline personality disorder among inner-city substance users: The role of childhood maltreatment, negative affect intensity/reactivity, and emotional dysregulation. Comprehensive Psychiatry, 49, 603-615.

Gross, J. (1998). The emerging field of emotion regulation: An integrative review. Review of General Psychology, 2, 271-299.

Gross, J. (1999). Emotion and emotion regulation. In L.A. Pervin y O.P. John. Handbook of personality: Theory and research (2nd ed.) (pp. 525-552). New York: Guilford Press.

Guzmán-González, M., Garrido, L. \& Leiva, J. (2014). Validez y Confiabilidad de la Versión Adaptada al Español de la Escala de Dificultades de Regulación Emocional (DERS-E) en Población Chilena. Terapia Psicológica, 32, 19-29.

Herrera, J., Niño, M.V., Caycedo, C. \& Cortés, O. (2008). Validación de la Escala de Desregulación Emocional en universitarios bogotanos. Tesis no publicada. Fundación Universitaria Konrad Lorenz.

Hervás G. \& Jódar, R. (2008). Adaptación al castellano de la Escala de Dificultades en la Regulación Emocional. Clínica y Salud, 19, 139-156.

Hirshfeld, R. \& Rusell, J. (1997). Assessment and Treatment of Suicidal Patients. The New England Journal of Medicine, 337, 910-915.

Jacobs, D. (1999). Depression screening as an intervention against suicide. Journal of Clinical Psychiatry, 60, 42-45.

Kököyei, G., Urbán, R., Reinhardt, M., Józan, A. \& Demetrovics, Z. (2014). The Difficulties in Emotion Regulation Scale: Factors Structure in Chronic Pain Patients. Journal of Clinical Psychology, 70, 589-600.

Lavender, J., Wonderlich, S., Engel, S., Gordon, K., Kaye, W. \& Mitchell, J. (2015). Dimensions of emotion dysregulation in anorexia nervosa and bulimia nervosa: A conceptual review of the empirical literature. Clinical Psychology Review, 40, 111-122.

Law, K. \& Chapman, A. (2015). Borderline personality features as a potential moderator of the effect of anger and depressive rumation on shame, self-Blame, and self-forgiveness. Journal of Behavior Therapy and Experimental Psychiatry, 46, 27-34.

León, D. (2006). ¿Es explicable la consciencia sin emoción?: Una aproximación biológico-afectiva a la experiencia consciente. Revista Latinoamericana de Psicología, 38, 361-381.

Linehan, M. (1993). Cognitive Behavioral Treatment of Borderline Personality Disorder._New York: Guildford Press.

Little, K., Welsh, D., Darling, N. \& Culpepper, C. (2015). Family Enmeshment, Adolescent Emotional Dysregulation, and the Moderating Role of Gender. Journal of Family Psychology, 29, 604-613.

Masi, G., Muratori, P., Manfredi, A., Pisano, S. \& Milone, A. (2015). Child behavior checklist emotional dysregulation 
profiles in youth with disruptive behavior disorders: Clinical correlates and treatment implications. Psychiatry Research, 225, 191-196.

Mann, J., Oquendo, N., Underwood, M. \& Arango, W. (1999). The neurobiology of suicide risk: A review for the clinician. Journal of Clinical Psychiatry, 60, 7-11.

Marin, M., Robles, R., González-Forteza, C. \& Andrade, P. (2012). Propiedades Psicométricas de la Escala "Dificultades en la Regulación Emocional" en español (DERS-E) para adolescentes mexicanos. Salud Mental, 35, 521-526.

McDermott, M. J., Tull, M. T., Gratz, K. L., Daughters, S. B. \& Lejuez, C. W. (2009). The role of anxiety and difficulties in emotion regulation in posttraumatic stress disorder among crack/cocaine-dependent patients in residential substance abuse treatment. Journal of Anxiety Disorders. 23, 291-299.

Ministerio de la Protección Social. (2005). Estudio Nacional de Salud Mental - Colombia 2003. Cali: C\&C Gráficas LTDA, Ministerio de la Protección Social- Fundación FES Social.

Medrano, L. A. \& Trógolo, M. (2014). Validación de la escala de dificultades en la regulación emocional en la población universitaria de Córdoba, Argentina. Universitas Psychologica, 13, 15-26

Moscicki, E. (1995). Epidemiology of Suicidal Behavior. Suicide and Life-Threatening Behavior, 25, 22-35.

Neacsiu, A., Eberle, J., Kramer, R., Wiesmann, T. \& Linehan, M. (2014). Dialectical behavior therapy skills for transdiagnostic emotion dysregulation: A pilot randomized controlled trial. Behavior Research and Therapy, 59, 40-51.

Posada-Villa, J., Aguilar-Gaxiola, S., Magaña, C. \& Gómez, L. (2004). Prevalencia de trastornos mentales y uso de servicios: resultados preliminares, estudio nacional de salud mental Colombia. Revista Colombiana de Psiquiatría, 23, 241- 262.

Powell, J., Geddes, J., Hawton, K., Deeks, J. \& Goldcare, M. (2000). Suicide in psychiatric hospital in-patients. British Journal of Psychiatry, 176, 266-272.

Powers, A., Stevens, J., Fani, N. \& Bradley, B. (2015). Construct validity of a short, self report instrument assessing emotional dysregulation. Psychiatry Research, 225, 82-92.

Rajappa K., Gallagher, M. \& Miranda, R. (2012). Emotion Dysregulation and Vulnerability to Suicidal Ideation and Attempts. Cognitive Therapy and Research. 36, 836-839.

Rich, C., Dhosse, D., Ghani, S. \& Isacsson, G. (1998). Suicide methods and presence of intoxicating absable substances: some clinical and public health implications. Annals of clinical Psychiatry, 10, 169-175.

Ridings, L. \& Lutz-Zois, C. (2014). Emotional Dysregulations and Borderline Personality Disorder: Explaining the link between secondary psychopathy and alexithymia. Personality and Individual Differences, 57, 14-19.

Roemer, L., Lee, J. K., Salters-Pedneault, K., Erisman, S.M., Orsillo, S. M. \& Mennin, S.M. (2009). Mindfulness and emotion regulation difficulties in General Anxiety Disorder: Preliminary evidence for independent and overlapping contributions. Behavior Therapy. 40, 142-154.
Ruganci, R. N. \& Gençöz, T. (2010). Psychometric properties of a Turkish version of the Difficulties in Regulation Emotional Scale. Journal of Clinical Psychology, 66, 442-455.

Salovey, P., Mayer, J. D., Goldman, S. L., Turvey, C. \& Palfai, T. P. (1995). Emotional attention, clarity and repair: Exploring emotional intelligence using the trait meta-mood scale. In J. W. Pennebaker (Ed). Emotion, disclosure, and health (pp. 125-154). Washington D.C.: APA Books.

Seligowski, A. \& Orcutt, H. (2015). Examining the Structure of Emotion Regulation: A Factor-Analytic Approach. Journal of Clinical Psychology, 71, 1004-1022.

Staples, A. \& Mohlman, J. (2012). Psychometric properties of the GAD-Q-IV and DERS in older, community-dwelling GAD patients and controls. Journal of Anxiety Disorders. 26, 385-392.

Stewart, I., Villatte, M. \& McHugh, L. (2012). Approaches to the self. In Louise McHugh and Ian Stewart (Eds). The self and perspective taking. Contributions and applications from modern behavioral science. Oakland: New Harbinger Publications.

Tull, M. T. (2006). Extending an anxiety sensitivity model of uncured panic attack frequency and symptom severity: the role of emotion dysregulation. Cognitive Therapy and Research, 30, 177-284.

Tull, M. T., Stipelman, B. A., Salters-Pedneault, K. \& Gratz, K. L. (2009). An examination of recent non-clinical panic attacks, panic disorder, anxiety sensitivity, and emotion regulation difficulties in the prediction of generalized anxiety disorder in an analogue sample. Journal of Anxiety Disorder, 23, 275-282.

Vargas, R. M., \& Muñoz-Martínez, A. M. (2013). La regulación emocional: precisiones y avances conceptuales desde la perspectiva conductual. Psicología-USP, 24, 225-240.

Weinberg, A. \& Klonky, E. D. (2009). Measurement of Emotional Dysregulation in Adolescents. Psychological Assessment, 21, 616-621.

Wehmeier, P. M., Schacht, A., \& Barkley, R. A. (2010). Social and emotional impairment in children and adolescents with ADHD and the impact on quality of life. Journal of Adolescent Health, 46, 209-217.

Weis, N., Gratz, K. \& Lavender, J. (2015). Factor Structure and Initial Validation of a Multidimensional Measure of Difficulties in the Regulation of Positive Emotions: The DERSPositive. Behavior Modification, 39, 1-23.

World Health Organization. (2012). The global burden of mental disorders and the need for a comprehensive, coordinated response from health and social sectors at the country level. Sixty-fifth world health assembly . Extraido de http://www. who.int

Zutphen, L., Siep, N., Jacob, G., Goebel, R. \& Arntz, A. (2015). Emotional sensitivity, emotion regulation and impulsivity in borderline personality disorder: A critical review of fMRI studies. Neuroscience and Biobehavioral Reviews, 51, 64-76. 